\title{
The Monash Spectrograph Simulation Program
}

\author{
S. C. Marsden ${ }^{1}$ and D. W. Coates \\ Department of Physics, Monash University, Clayton, Vic. 3168, Australia \\ dcoates@sci.monash.edu.au \\ ${ }^{1}$ Present address: CSIRO Division of Atmospheric Research, Aspendale, Vic. 3195, Australia \\ scm@larry.dar.csiro.au \\ Received 1997 June 12, accepted 1997 August 21
}

\begin{abstract}
The Monash University Physics Department is constructing a spectrograph, to be attached to a $0 \cdot 46-\mathrm{m}$ Cassegrainian telescope. To help future users of the spectrograph determine the operational capabilities of the spectrograph a PC-based software package was created. This program allows the user to simulate the response of the spectrograph to various stellar types under differing observational constraints. We have estimated the precision of measurements of stellar radial velocities with the spectrograph. The estimates are reasonable but are yet to be compared with real data.
\end{abstract}

Keywords: instrumentation: spectrographs

\section{Introduction}

The Monash observatory, situated approximately $50 \mathrm{~km}$ East-South-East of Melbourne at an elevation of $308 \mathrm{~m}$, is currently used for differential photometric studies of rapidly rotating, active-chromosphere, cool stars. To expand the range of studies at the observatory, the Physics Department plans to attach a visible-wavelength spectrograph to its $0 \cdot 46$ $\mathrm{m}$ Cassegrainian reflector. This spectrograph is of a crossed Czerny-Turner design that is to operate in the visible wavelength band ( $3000 \AA$ to $7000 \AA)$. The CCD detector consists of two arrays of pixels with each array containing 2048 elements; each pixel is $13 \mu \mathrm{m}$ wide by $750 \mu \mathrm{m}$ high. It will operate at $-50^{\circ} \mathrm{C}$. The spectrograph itself has a linear reciprocal dispersion of $40 \AA$ per $\mathrm{mm}$ at $5000 \AA$ and will primarily be used to determine the radial velocities of the stars in the Monash observing program.

To enable users of the spectrograph to estimate the precision of radial velocity measurements acquired with the spectrograph, the Monash Spectrograph Simulation Program (MSSP) was created (Marsden 1996). The MSSP is a PC-based software package written in Fortran that allows the user to simulate the effect of the telescope, spectrograph and atmospheric conditions on spectra from various cool star types. The MSSP will also help users to determine the best operational parameters to be used during actual observations with the spectrograph, depending upon the star being studied and the atmospheric conditions during the observation. We outline below how the MSSP simulates the stellar and atmospheric variables involved in observing spectra. We also compare the MSSP's theoretical data with theoretical data from other spectrographs. Also outlined are the expected precisions in the radial velocity measurements to be taken with the spectrograph.

\section{Creation and Operation of the MSSP}

\subsection{Creation of an Ideal Spectrum}

To produce simulated spectral observations, the MSSP requires the user first to synthesise ideal normalised spectra of various stellar types. We have used the University of California at Los Angeles SYNthesis code (UCLASYN) (Dworetsky \& Jacobs 1985; Etzel 1982) for this purpose; line lists and oscillator strengths were taken from Thévenin (1989, 1990). The synthesised spectra were then modified by the MSSP for the observational variables listed below, to produce an ideal noise-free spectrum. The method used by the MSSP is outlined below.

Rotational broadening: This is simulated in the MSSP by convolving the spectrum being studied with a rotational broadening curve based on the star's projected rotational velocity $v \sin (i)$. Other broadening effects are assumed to be minimal, especially as most of the stars in the Monash observing program are rapid rotators.

Blackbody curve: The spectra produced by UCLASYN have a normalised continuum level. To have the spectrum more representative of an actual stellar spectrum, the MSSP puts the spectrum into a blackbody form based on the effective temperature of the star.

Doppler shift: The spectrum is Doppler shifted in wavelength by the MSSP for the chosen line-of-sight radial velocity. 
Atmospheric scattering: The effect of Rayleigh scattering in the atmosphere is simulated by reducing the spectrum's flux using the equation

$$
F_{B \lambda}=F_{T \lambda} 10^{-0 \cdot 4 K_{\lambda}},
$$

where

$$
K_{\lambda}=\left(0 \cdot 06+0 \cdot 00752 \lambda^{-4}\right) \sec (z) .
$$

Here $F_{T \lambda}$ is the spectrum's flux at the top of the Earth's atmosphere, $F_{B \lambda}$ is the spectrum's flux at the bottom of the Earth's atmosphere, $\lambda$ is the wavelength in microns, and $z$ is the star's zenith angle. The factor $\sec (z)$ assumes that the Earth's atmosphere is flat and is reasonably valid for zenith angles less than about $60^{\circ}$ (Hardie 1962).

Mirror reflectivity: The reflectivity of each of the four mirrors in the telescope and spectrograph was taken to be $73 \%$ at all visible wavelengths studied. This assumes a basic reflectivity of $80 \%$ reduced over a year by unavoidable deterioration. See Walker (1984) for data on dust build-up on mirrors at the South African Astronomical Observatory.

Slit-width: The throughput of the Monash spectrograph's entrance slit was calculated using data from the Royal Greenwich Observatory (RGO) spectrograph of the Anglo-Australian Observatory (Robinson 1985). These data give the percentage slit throughput for a given slit-width for four fullwidth at half-maximum (FWHM) seeing disks $(0 \cdot 5$, $1 \cdot 0,2 \cdot 0$ and $5 \cdot 0 \operatorname{arcsec})$. To convert this to the Monash spectrograph slit-width, the plate scales of the two spectrographs were used. As the data are given for only these four FWHM values and given that a seeing of 0.5 arcsec is very unlikely at our observatory, the MSSP simulates only the latter three values of seeing.

Instrumental profile: This is simulated by convolving the spectrum being studied with a gaussian based on the spectrograph's coma, spherical aberration, linear reciprocal dispersion, and on the chosen slit-width.

Grating sensitivity: For this simulation the MSSP uses data from the manufacturer and assumes that the incident light is polarised equally parallel and perpendicular to the grating's grooves.

Detector sensitivity: The sensitivity of the CCD detector used in the Monash spectrograph was supplied by the CCD manufacturer and is given as the quantum detection efficiency $Q_{D}$ of the CCD, which is based upon the detector's signal-to-noise ratio (SNR):

$$
Q_{D}=\frac{(\mathrm{SNR})_{\mathrm{OUT}}^{2}}{(\mathrm{SNR})_{\mathrm{IN}}^{2}}
$$

However, the actual efficiency required for the MSSP is the probability that any given photon will be detected by the CCD as a function of wavelength. This is called the quantum efficiency $Q$ of the detector:

$$
Q=\frac{N_{\text {EVENTS }}}{N_{\text {PHOTONS }}}
$$

If we assume that the CCD detector is ideal, then

$$
Q_{D}=\left(\frac{\sqrt{N_{\text {EVENTS }}}}{\sqrt{N_{\text {PHOTONS }}}}\right)^{2}=Q .
$$

This has been assumed to be the case in the MSSP, which is a reasonable approximation. However, $Q_{D}$ is usually less than $Q$.

Rebinning: Once all the previously mentioned effects have been simulated, the MSSP rebins the spectrum into the pixel size of the CCD. The relative flux at each pixel is calculated to produce a normalised noise-free spectrum.

\subsection{Creation of a Simulated Spectrum}

To convert the normalised noise-free spectrum into one likely to be observed with the Monash spectrograph, the MSSP must calculate the number of photons detected by the CCD and the amount of noise during each exposure. To calculate the number of photons detected by the CCD, the average flux arriving at the top of the Earth's atmosphere is used (Allen 1973). This is converted to the flux detected by the CCD by the processes outlined in Section 2.1, and thus into the average number of photons detected by the CCD from a star of given visual magnitude.

Because the actual number of photons arriving in any given time is random, the average number of photons calculated above is put through a Poissonian distribution before each exposure. This gives the actual number of photons detected by the CCD during each exposure. Then, using the noise-free spectrum obtained after rebinning as a probability template, the photons are randomly 'fired' at the CCD, using a uniform distribution to determine the pixel number. The chance of being detected by the CCD is based upon the intensity of the noisefree spectrum at the corresponding pixel. This is continued until all the photons have been detected by the CCD. This creates a photon spectrum containing only photon noise, to which must still be added the non-statistical noise. The two types of non-statistical noise simulated by the MSSP are background light and the electrical noise produced by the CCD and its associated electronics.

In order to simulate the background noise in the spectrum, the MSSP uses data based upon photometric data taken at the site, as there are no other data available. Thus the background light is only an estimate and contains no information on 
additional spectral lines or the dependence of the intensity of the background light upon wavelength. The background light added to the spectrum by the MSSP can have one of three levels: dark sky (new moon), average sky (quarter moon), and bright sky (full moon).

The second source of non-statistical noise in the spectrum, from the CCD and its associated electronics, was calculated from data on the electronic noise of the CCD (Coutures \& Boucharlat 1987) and an estimate of the off-chip noise of the electronics.

Once the amount of non-statistical noise is calculated, from both the background light and the CCD noise, both are converted to electron numbers and are each randomised by a Poissonian distribution before each exposure, similar to that done to the photon numbers, to represent the random nature of the noise. The electrons are then distributed randomly through the photon spectrum by using a uniform distribution. This then produces a simulation of a spectrum similar to that produced by the Monash spectrograph.

This simulated spectrum can then be crosscorrelated with a spectrum from a comparison star to give a simulated radial velocity measurement.

\section{Comparison of Results}

As mentioned previously, the results from the MSSP were to be compared with actual results from the completed Monash spectrograph. However, the spectrograph is still to be completed so it has been impossible to do comparisons. However, the theoretical data from the MSSP have been compared with other data, both theoretical and actual, from other spectrographs.

\subsection{Throughputs and Count Rates}

A comparison of the theoretical throughput of the Monash spectrograph was made with the theoretical throughput of the Faint Object Red Spectrograph (FORS) on the Anglo-Australian Telescope. Also, a comparison was made between the theoretical count-rate of the Monash spectrograph with that of the RGO spectrograph assuming both were situated on the Monash telescope.

As the components of the FORS and the Monash spectrograph have similar overall transmittance, one expects the spectrograph's theoretical peak system efficiencies also to be similar. This was found to be so: the theoretical peak system efficiency of the FORS is $11 \cdot 4 \pm 0 \cdot 2 \%$ (Couch 1989) and the theoretical peak system efficiency of the Monash spectrograph, calculated by the MSSP, is $11 \cdot 3 \pm 0 \cdot 1 \%$.

We compared the calculated theoretical countrate for a star of a particular visual magnitude using the RGO spectrograph, assuming that it is situated on the Monash telescope, with the count-rate predicted by the MSSP for the Monash spectrograph. These count-rates should be similar, because the spectrographs' components are similar. For a star of $V=8$ and based on the count-rates given in Sadler, Harrison \& Lee (1991), a theoretical count-rate of 42 counts per $\AA$ per second at $5000 \AA$ for the RGO spectrograph on the Monash telescope was calculated. For a star of this visual magnitude the MSSP predicts 38 counts per $\AA$ per second at $5000 \AA$ for the Monash spectrograph.

We believe that these comparisons indicate that the calculations by the MSSP are reasonable.

\subsection{Radial Velocity Measurements}

The main purpose of the Monash spectrograph is to determine stellar radial velocities. To determine the precision of expected radial velocity measurements, several simulations of target and comparison stars were made. A sample target star was of type K0V, with a visual magnitude $m_{V}=11 \cdot 0$ and a simulated projected rotational velocity $v \sin (i)$ of $50 \mathrm{~km} \mathrm{~s}^{-1}$. The standard comparison star simulated by the MSSP was a $\mathrm{K} 2 \mathrm{~V}$ type star with a visual magnitude of $m_{V}=4.0$ and a simulated projected rotational velocity $v \sin (i)$ of $50 \mathrm{~km} \mathrm{~s}^{-1}$. Exposure times were 1000 and $100 \mathrm{~s}$ for the target and comparison star respectively.

To simulate a radial velocity measurement, the MSSP was used to simulate nine separate exposures of the target star and one exposure of the comparison star. Only one exposure of the comparison star was taken, as this star is relatively bright and the variations between exposures were shown to have little effect on the estimation of the radial velocity measurements. The nine exposures of the target star were cross-correlated with the exposure of the comparison star using the FIGARO spectral analysis code (Shortridge 1991). The standard deviation ( $1 \sigma$ value) of these nine measurements was used to estimate the uncertainty in the radial velocity measurements. For the standard comparison and target stars listed above, the standard deviation of the nine radial velocity measurements is $5.5 \mathrm{~km}$ $\mathrm{s}^{-1}$.

As there are no actual data from the Monash spectrograph, we have compared the above results with data for three stars in the Monash observing program, AB Doradus (HD 36705), PZ Telescopium (HD 174429), and CF Octantis (HD 196818), observed by a former Monash PhD student (Innis 1986). The radial velocities of these stars were taken with the Boller and Chivens echelle spectrograph at the Cassegrainian focus of the $1 \cdot 0-\mathrm{m}$ telescope at the Siding Spring Observatory, or with the coudé echelle spectrograph on the $1.9-\mathrm{m}$ telescope at the Mt Stromlo Observatory.

The three real stars are all late-type stars similar to the sample target star simulated by the MSSP. The $1 \sigma$ values for the real stars are similar to 
those for the simulated star. As the real stars were observed with SNRs of around 10:1 to $30: 1$, and the SNR for the simulated star is approximately $25: 1$, this would seem to lend some support to the accuracy of the simulated results given here, even though the spectra from the three real stars were taken with spectrographs of higher spectral resolution than the Monash spectrograph.

\section{Limitations and Further Work}

Although the MSSP can simulate spectra from the Monash spectrograph, it still has some limitations. The main limitation is that it simulates only the Monash spectrograph. However, the MSSP could be adapted easily to simulate other spectrographs. Also, the current version of the MSSP uses routines for plotting the spectra studied that rely upon a graphics package called HALO professional ${ }^{1}$. As these subroutines are subject to copyright they are not included with the MSSP. However, if a user has a copy of HALO professional then the plotting routines will work normally; if not, then the spectra are stored in ASCII files so it should be possible to plot them with virtually any plotting program. Further information on the MSSP, as well as the latest version of the program, can be obtained from the authors.

\section{Conclusions}

Although the comparative observed data for the MSSP's simulations are limited, the data available indicate that the MSSP's estimation of the precision of radial velocity measurements is reasonable. However, the true test of its abilities will come when the Monash spectrograph is completed and measurements can be made with it. If the comparison of the MSSP's simulated results and that of the actual results turn out to be favourable, it will be of use to future users of the Monash spectrograph and, with some

$\overline{1}$ HALO professional is produced by Media Cybernetics, Maryland, USA. modifications, be of value to the users of other spectrographs.

\section{Acknowledgments}

The authors would like to acknowledge the help of Colin Porter (formerly of Monash University) for his help with the technical details of the Monash spectrograph, and Dr Richard Austin (CSIRO Division of Atmospheric Research) for his help in proofreading not only this paper but S. C. Marsden's Masters thesis. He was not properly acknowledged for this in the thesis and the authors would like to do so now.

Allen, C. W. 1973, Astrophysical Quantities, 3rd edn (London: Athlone Press)

Couch, W. 1989, FORS Users Manual (Epping: AngloAustralian Observatory)

Coutures, J. L., \& Boucharlat, C. 1987, A 2×2048 Pixel Bilinear CCD Array for Spectroscopy (Boulogue: ThomsonCSF)

Dworetsky, M. M., \& Jacobs, J. M. 1985, Astronomers Guide to Spectrum Synthesis and Curve of Growth Analysis with UCLA Spectrum Synthesis program (London: University College London)

Etzel, P. B. 1982, The J. E. Ross Stellar Spectrum Synthesis Code: Kludge Documentation (Los Angeles: Astronomy Department UCLA)

Hardie, R. H. 1962, in Astronomical Techniques. Stars and Stellar Systems, Vol. 2, ed. W. A. Hiltner (Univ. Chicago Press), 178

Innis, J. L. 1986, A study of several late-type very activechromosphere stars, $\mathrm{PhD}$ Thesis, Monash University

Marsden, S. C. 1996, Simulation of the Monash Spectrograph: A study of the radial velocities of cool stars, MSc Thesis, Monash University

Robinson, R. D. 1985, The RGO Spectrograph, 2nd edn (Epping: Anglo-Australian Observatory)

Sadler, E., Harrison, S., \& Lee, S. 1991, Anglo-Australian Observatory: Observers Guide, 4th edn (Epping: AngloAustralian Observatory)

Shortridge, K. 1991, Figaro Release Notes-Version $3 \cdot 0$ (Epping: Anglo-Australian Observatory)

Thévenin, F. 1989, A\&A Suppl. Series, 77, 137

Thévenin, F. 1990, A\&A Suppl. Series, 82, 179

Walker, A. R. 1984, MNRAS, 209, 83 